Projets

de paysage

\section{Projets de paysage}

Revue scientifique sur la conception et l'aménagement de l'espace

$8 \mid 2012$

Les concepteurs de jardins et de parcs japonais

\title{
Les modalités de gestion et d'entretien des parcs publics en Tunisie
}

Le cas de la région de Tunis

Public Parks and gardens management and maintenance regimes - The case of

Tunis, Tunisia

\section{Besma Loukil, Taoufik Bettaieb et Pierre Donadieu}

\section{OpenEdition \\ Journals}

Édition électronique

URL : https://journals.openedition.org/paysage/15969

DOI : $10.4000 /$ paysage. 15969

ISSN : 1969-6124

\section{Éditeur :}

École nationale supérieure du paysage de Versailles-Marseille, Institut national des sciences appliquées Centre Val de Loire - École de la nature et du paysage, École nationale supérieure d'architecture et de paysage de Bordeaux, École nationale supérieure d'architecture et de paysage de Lille, Agrocampus Angers

\section{Référence électronique}

Besma Loukil, Taoufik Bettaieb et Pierre Donadieu, «Les modalités de gestion et d'entretien des parcs publics en Tunisie », Projets de paysage [En ligne], 8 | 2012, mis en ligne le 13 juillet 2012, consulté le 07 décembre 2022. URL : http://journals.openedition.org/paysage/15969 ; DOI : https://doi.org/10.4000/ paysage. 15969

Ce document a été généré automatiquement le 11 mars 2021.

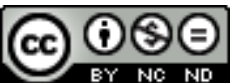

Creative Commons - Attribution - Pas d'Utilisation Commerciale - Pas de Modification 4.0 International - CC BY-NC-ND 4.0

https://creativecommons.org/licenses/by-nc-nd/4.0/ 


\title{
Les modalités de gestion et d'entretien des parcs publics en Tunisie
}

\author{
Le cas de la région de Tunis \\ Public Parks and gardens management and maintenance regimes - The case of \\ Tunis, Tunisia
}

Besma Loukil, Taoufik Bettaieb et Pierre Donadieu

1 Les parcs et les jardins publics dans les pays du Maghreb ont été originellement aménagés dans les villes par la colonisation française et répondent à des enjeux d'hygiène et d'esthétique publics de ces villes. Après des décennies d'urbanisation de la capitale et des grandes villes, une politique publique nationale de création d'espaces verts publics a vu le jour en 1991 en Tunisie. Cette politique a émergé sous des thématiques diverses mais essentiellement liées au développement durable, à l'environnement, à l'esthétique et à la propreté urbaines. En dépit des réalisations effectuées sur tout le territoire tunisien, ces espaces sont souvent peu entretenus, connaissent un manque de satisfaction, voire un non-retour des usagers (Loukil, 2006). L'état de ces parcs, l'augmentation de certains comportements perçus comme incivils, le sentiment d'insécurité, la situation géographique de leurs territoires d'inscription et le contexte socio-économique des quartiers environnants sont des causes de cette insatisfaction. Ces dernières ainsi que l'état d'entretien varient donc d'un parc à un autre.

2 Pourquoi certains parcs ou jardins bénéficient-ils d'un travail régulier et intensif alors que d'autres semblent délaissés? S'agit-il de facteurs liés aux caractéristiques de ces espaces ou plutôt aux modalités de régie? Afin de répondre à ces interrogations nous avons travaillé sur un échantillon de 76 espaces verts publics dans un rayon de 60 kilomètres autour de Tunis. Nous avons élaboré une base de données les caractérisant. Les espaces inventoriés ont été classés selon leur état, variant de « en bon état » à « en état médiocre». Des entrevues ont été réalisées non seulement auprès des 
gestionnaires de ces espaces mais aussi auprès des décideurs institutionnels responsables de leur création et de leur administration. Une étude plus approfondie de trois parcs dans la région de Tunis a concerné les jardiniers et les agents de gardiennage et nous a donné des précisions sur les différentes modalités de gestion présentes dans la région de Tunis et probablement représentatives des stratégies utilisées dans le territoire national.

Les recherches faites sur le maintien technique des espaces verts urbains en général (Bretzel et al., 2010), et les parcs et les jardins publics en particulier, traitent des questions comme la nature du sol, le compostage, la maitrise de l'irrigation et le choix d'espèces locales, etc. (Joshi et al., 2010). Des recherches sur les politiques publiques et sur les acteurs ayant la responsabilité de gérer et de maintenir les espaces verts urbains se font plus rares (Barthe-Deloisy, 1997). En effet, il y a une documentation limitée sur le thème de la gestion des parcs et des jardins publics même dans les pays du Nord (Randrup et al., 2009). Les études sur le contrôle social formel (Morgan et al., 1995) informel (Nugier, 2007) dans l'espace public ont été faites également dans les pays du Nord mais pas encore dans les pays du Sud. Dans le contexte d'un pays en voie de développement comme la Tunisie, l'État s'occupe, grâce à des institutions publiques et des entreprises privées (bureaux d'études, concepteurs, entrepreneurs, etc.), de l'étude, de la conception, de l'exécution et de la gestion de ces espaces. Des études ont été faites en Tunisie sur les ambiances urbaines d'une place publique (Ben Ayed-Najjar, 2007), sur l'intégration urbaine d'un jardin public (Ben Jbara, 2006) ou sur les circonstances et le contexte historique de l'installation d'un parc (Zaâfrane-Zhioua, 1998). Ont été également analysés les possibilités de faire revivre ces espaces (Zahaf, 1999), la programmation des espaces verts, leurs rapports aux documents d'urbanisme et à la planification urbaine (Turki et al., 2009), ainsi que les pratiques observées dans un parc universitaire (Jaballah, 2006), etc. La recherche faite par nous-mêmes depuis 2008, dans le cadre de notre travail de thèse ${ }^{1}$, apporte des éclairages sur l'explication des comportements des usagers en fonction du mode de fonctionnement de ces espaces. Cet article, qui exprime une partie des résultats de notre thèse, se limite à l'étude de la question de la gestion des parcs et des jardins publics.

\section{La gestion, l'entretien et le contrôle : des outils de prévention contre le mauvais fonctionnement de l'espace public}

$4 \quad$ Les parcs et les jardins publics sont des espaces urbains ouverts au public, en général fortement régulés par les gestionnaires; ils présentent des modes qui varient cependant entre l'hyporégulation: avec abandon relatif et apparitions d'incivilités, et l'hyperrégulation pouvant aboutir à l'exclusion, à la sélection et à la privatisation des usages (Donadieu, 2007). En Tunisie, il y a des parcs libres d'accès avec ou sans clôtures, d'autres clôturés et qui ferment leurs portes la nuit, des parcs publics à usage public et d'autres à usage privatif. Dans chacun de ces cas, la gestion et le fonctionnement qui en découle sont différents. En ce qui concerne l'espace public, nous ne pouvons parler ni de productivité ni de rentabilité au sens économique, mais de fonctionnalités matérielles et d'usages sociaux. 


\section{Nécessité d'un entretien et d'un contrôle}

5 L'entretien correspond aux soins, réparations et dépenses qu'exige le maintien de quelque chose ou d'un patrimoine en bon état. Un ensemble de fonctions dérive de cette notion : équipe, agent (technicien, ouvrier, etc.), service d'entretien... (Nouveau Petit Robert, 2001). Le terme signifie l'organisation nécessaire pour mener à bien les projets, et prévenir des anomalies ou défauts graves qui rendent une chose inutilisable ou empêchent son bon fonctionnement. Nous entendons en effet par cette expression la prévention, c'est-à-dire une action positive: mieux vaut empêcher qu'un fait se produise que d'en réparer les résultats néfastes. Le mot dérive du verbe " gérer » c'està-dire contrôler financièrement, organiser, donner en détail la synthèse de la situation à un moment donné et maîtriser par des moyens appropriés à chaque situation. C'est aussi faire bien fonctionner. La gestion suppose également d'améliorer les performances d'un système d'actions existant en fonction d'objectifs explicites posés au départ (Donadieu, Mazas, 2002). Le système d'actions regroupe ainsi les acteurs et le contexte de leurs pratiques sociales. La gestion publique relève, en principe, des autorités publiques (municipalités, ministères, etc.) qui décident des projets et de leur évolution. En réalité, les espaces publics, en général, résultent d'une négociation permanente entre le secteur privé et le secteur public (Ghorra-Gobin, 2000). Entretenir l'espace public, des parcs et des jardins en particulier, s'avère un «acte moral » des gestionnaires vis-à-vis de la société civile et non pas une simple tâche technique quotidienne.

6 En outre, le contrôle des parcs et des jardins publics les protège en principe contre tous types de pratiques portant atteinte au bon état des aménagements et des équipements existants. Contrôler c'est aussi maîtriser, surveiller et empêcher les dérives (Boudon et Bourricaud, 2004).

7 Lorsqu'on parle de l'usage de l'espace public, on ne peut pas écarter la question du contrôle social, formel et informel. Le sociologue québécois Robert Campeau précise les diverses formes du contrôle social. Le « contrôle social formel » est le respect de la loi et de l'ordre par les représentants de la police judiciaire (policiers, juges, avocats et militaires) ou par l'idéologie dominante. En ce sens le contrôle social formel permet de faire respecter la loi et l'ordre par les autorités publiques. Le "contrôle social informel » correspond aux pressions indirectes sur l'individu, c'est-à-dire d'une façon subtile dans son milieu de vie immédiat (Campeau et al., 1998).

Puisque les comportements des usagers peuvent aller à l'encontre des normes sociales, la présence des individus - peu importe leurs statuts : usagers ou gestionnaires de l'espace - est essentielle dans l'espace public urbain. Car contrôler c'est aussi maîtriser. Dans la sociologie américaine, le contrôle social est évoqué dans les études concernant la déviance et la criminalité. Le contrôle social prend un aspect positif, celui de la régulation: la contrainte externe devient donc interne et prend la forme d'une obligation morale comme l'affirme le sociologue Talcott Parsons. (Parsons, 1998). Contrôler, c'est notamment surveiller et empêcher (Boudon et Bourricaud, op.cit.). D'après le Dictionnaire de l'urbanisme et de l'aménagement, ce terme peut être défini comme « l'ensemble des sanctions positives et négatives auxquelles une société recourt pour assurer la conformité des conduites aux modèles établis » (Cusson, 1983, cité par Merlin et Choay, 2005). 


\section{Répartition spatiale des parcs et des jardins publics et inégalités dans leur fonctionnement et contrôle au Nord-Est de la Tunisie}

Les parcs et les jardins publics urbains sont inégalement répartis dans les villes du Nord-Est tunisien. La majorité des espaces étudiés est localisée dans la ville de Tunis, avec une concentration dans les délégations renfermant des quartiers aisés ; c'est le cas par exemple de la délégation Menzah située au Nord-est de Tunis. Les quartiers populaires sont en revanche démunis ou disposent d'une surface beaucoup plus faible d'espaces verts.

L'étude de la répartition des parcs au Nord-Est de la Tunisie a montré que cette politique des parcs et des jardins publics a été marquée par des différences importantes au niveau de la création, de la gestion et du contrôle de ces espaces. Alors que dans les quartiers populaires, où les conditions socio-économiques sont plus difficiles, les espaces verts publics sont plutôt délaissés ${ }^{2}$, dans les quartiers aisés ils sont en général plus denses, bien entretenus et gardés, surtout lorsque ces quartiers ont une importance politique ou économique (figure 1).

Figure 1. Répartition des différents modes de gestion ${ }^{3}$ selon l'environnement urbain dans lequel se situe le parc

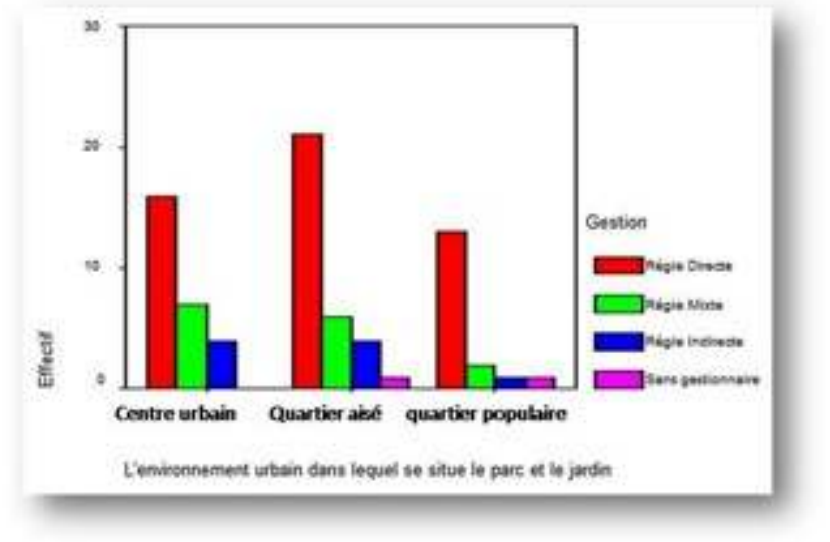

Source : Besma Loukil, 2009.

11 Ceci s'explique par le mode de gérance qui est en régie mixte : à la fois par l'État et par une entreprise privée. Ce mode est localisé dans les quartiers aisés et les centres urbains. Le mode de régie directe est dominant dans le Nord-Est de la Tunisie est celui assuré par les municipalités.

\section{L'état des parcs comme résultat de leur administration}

12 L'état des parcs et des jardins publics dépend tout d'abord des moyens mis en œuvre par les gestionnaires pour maintenir les parcs et les jardins loin des dégradations des équipements, des espaces jardinés. Ensuite, il dépend aussi du moment et du mode d'intervention. Et en particulier du fait que les réparations sont faites rapidement ou tardivement. Dans ce cas de figure les chercheurs américains Wilson et Killing ont développé une théorie appelée théorie de la vitre brisée qui indique que si une vitre dans 
un immeuble n'est pas rapidement réparée, le reste des vitres sera rapidement dégradé car cela signale un lieu sans loi (Wilson et Kelling, 1982). Enfin, le maintien en bon état de l'espace public se fait à travers la présence continue de ces usagers et dépend de la manière avec laquelle l'espace a été conçu: la théorie de l'espace défendable (Newman, 1972 ; Jacob, 1961). Il s'agit d'une théorie qui se base sur deux aspects. Le premier est la présence effective des usagers. Le second concerne l'aménagement de l'espace, qui se fait de manière à assurer la sécurité et la lisibilité de l'espace. C'est une notion relative au « maintien de l'ordre » et au contrôle informel.

13 L'étude des parcs et des jardins publics au Nord-Est de la Tunisie a confirmé ces deux théories. Elle a montré que rares sont les cas où les réparations sont vite exécutées et que l'état dépend non seulement des facteurs susmentionnés, mais aussi d'autres causes géographiques et de facteurs spécifiques au contexte politique de la Tunisie. Il s'agit de parcs proches des résidences présidentielles et qui disposent d'un suivi régulier. C'est le cas par exemple des parcs et des jardins situés dans le village de Sidi Bou Saïd (parc de Sidi Bou Saïd et Jardin méditerranéen) et Carthage (parc Al-Abidine). Dans ce cas les dégradations sont vite réparées. Alors que dans le parc Kram, un cas assez représentatif de la majorité des parcs et des jardins gérés par les municipalités les dégradations sont peu réparables.

14 La fidélisation des usages et le maintien de certaines catégories d'usagers comme les familles et les sportifs contribuent à assurer, par leur présence, la sécurité de l'espace. C'est ce qu'Oscar Newman appelle «la présence effective des usagers». Un espace fréquenté est un espace qui inspire la sécurité. En l'absence d'usagers, des situations de désordre s'installent comme l'indique un cadre du ministère de l'Environnement et du Développement durable (MEDD), en parlant d'un parc en cours d'exécution : «Vous ne pourrez jamais imaginer la quantité de bouteilles d'alcool qu'on a trouvées dans cet espace. C'est une région très dangereuse. Lors de la visite de ce terrain nous étions accompagnés par le délégué de la région, et c'était très dangereux comme espace. L'emplacement de ce futur parc est très important. C'est un belvédère. Il pourrait constituer un bon parc. Déjà les groupes qui pratiquent l'espace l'ont déserté parce qu'ils ne pourraient plus rien faire en la présence de nos agents et avec le commencement des travaux d'aménagement. Dès qu'on attribue une fonction à un espace, les autres s'éloignent parce qu'ils n'y trouvent plus leur compte. Ils seront gênés et ne pourraient plus se comporter librement. Après l'ouverture du parc au public, il y aura des familles qui viendront y passer du temps. La présence de ces familles empêchera les autres d'y venir. Parce que les autres cherchent l'isolement, pour pratiquer ce qu'ils veulent. C'est ça la sécurité."

Ces questions concernent tous les niveaux: la conception, les espèces végétales utilisées, les équipements publics, les matériels, la présence ou l'absence de fontaines fonctionnelles ou d'espaces fleuris, le nombre d'ouvriers et de gardiens, etc. Ces différences participent à la distinction de milieux sociaux de vie, et parfois à l'augmentation de l'exclusion sociale et des disparités territoriales dans les différents tissus de la ville.

\section{Ce que disent les gestionnaires des espaces verts}

La gérance des parcs et des jardins publics en Tunisie est assurée en général par l'État ( $86 \%)$, alors qu'une majorité (66\%) est assurée par les municipalités (régie directe) c'est-à-dire sous la tutelle du ministère de l'Intérieur et du Développement local. 
Certains, qui sont très rares, sont gérés par le ministère de l'Environnement ou les municipalités avec une entreprise privée (régie mixte : $20 \%$ ). D’autres municipalités, par le biais de conventions, donnent la gestion à des entreprises privées et assurent à des degrés moindres le suivi des travaux (régie indirecte : $12 \%$ ). D'autres parcs sont gérés par l'Agence nationale de propreté de l'environnement, sous la tutelle du ministère de l'Environnement, mais sont en régie directe parce qu'ils sont gérés par des fonctionnaires de l'État.

17 L'insuffisance des budgets nécessaires au maintien des espaces verts est citée par la majorité des acteurs municipaux comme la principale raison expliquant les problèmes de qualité de ces espaces et des incivilités qui en dérivent. En effet comme le souligne un chef de service au sein du ministère de l'Environnement et du Développement durable, il y a, d'une part, une disparité flagrante dans la répartition des budgets municipaux et, d'autre part, des budgets minimes qui sont loin d'assurer les tâches indispensables au maintien de ces espaces dans un bon état.

\begin{abstract}
« À titre d'exemple, le budget de la municipalité de Zahra est de 15000 DT, ce qui ne couvre même pas les frais d'une étude. Le budget destiné à la gestion des espaces verts pour les municipalités est très faible. Le budget alloué aux espaces verts dans les municipalités atteint idéalement le $1 \%$. Les municipalités sont débordées. Elles ne peuvent pas assurer l'entretien indispensable au maintien de leurs espaces. À mon avis il faut léguer ces espaces au privé... Le parc du Kram est administré actuellement par la municipalité, dont le budget consacré aux espaces verts est très faible. Les municipalités ont d'autres priorités de propreté et autres que la création des espaces verts. »
\end{abstract}

Les municipalités des zones populaires disposent de budgets faibles par rapport à ceux des zones résidentielles, et la part allouée au maintien des jardins publics est parfois inexistante. Dès lors, la probabilité de dégradation est grande puisque les services municipaux n'ont pas les moyens pour accomplir les tâches nécessaires, ou ont d'autres priorités (figure 2).

Régenter les espaces verts s'avère une opération coûteuse qui dépasse largement les budgets municipaux ${ }^{4}$. Ces derniers sont le plus souvent insuffisants pour assurer les opérations de gestion.

C'est aussi le cas des jardins du programme de réhabilitation des quartiers populaires, créés par le ministère de l'Environnement et donnés ensuite aux municipalités pour assurer la gestion. «[...] les autres parcs qui sont managés par les municipalités n'ont pas de cadre de suivi... Ils ne font l'objet d'aucune planification dans ce sens. Les parcs et les jardins qui sont régis par les municipalités sont laissés à leur propre sort. » (Entretien avec un ingénieur au MEDD.) 
Figure 2. Jardin public en très mauvais état dans le quartier populaire Ouardia (Tunis)

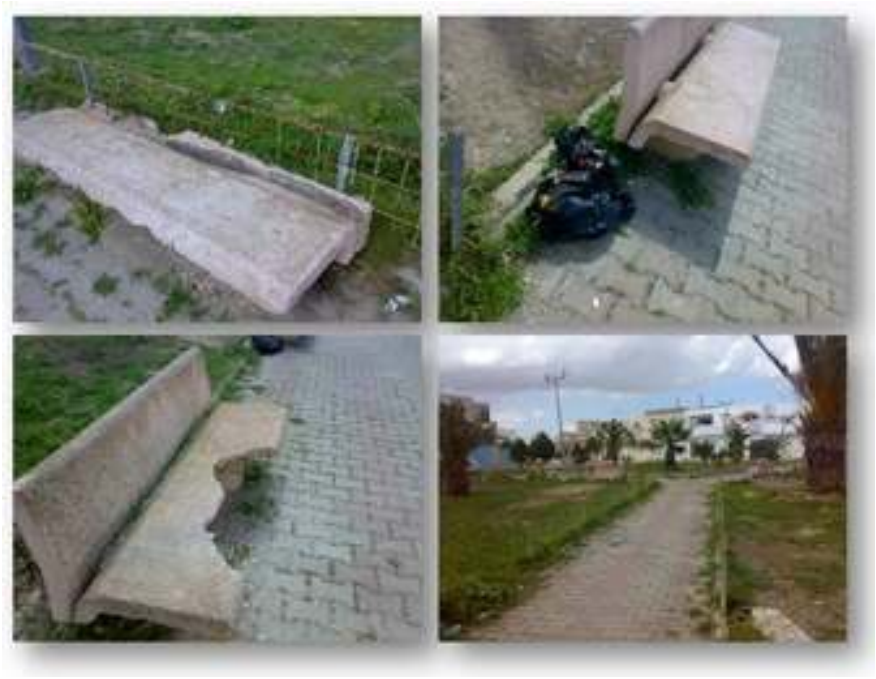

Source : Besma Loukil.

pas dotés des mêmes stratégies. Il y a ceux managés par le ministère de l'Environnement et ceux dirigés par l'Agence nationale de protection de l'environnement, et une majorité administrée par les municipalités. Certains parcs sont en régie mixte où les entreprises privées signent des conventions avec les municipalités ou le ministère.

\section{Ce qu'apporte la gestion assurée par l'État}

Les parcs et les jardins dirigés par le ministère de l'Environnement (figure 3) et par l'Agence nationale de protection de l'environnement sont dotés d'avantages importants. Ces parcs disposent de budgets qui leur sont propres et bénéficient de marchés-cadres, sorte de conventions avec des entrepreneurs privés. Pour les parcs du ministère de l'Environnement :

«[...] les parcs que nous avons nous-même aménagés, Sidi Bou Said et El-Abidine, font l'objet d'un soin particulier. En effet, ces deux parcs bénéficient d'un marchécadre. Il s'agit du maintien de tout ce qui est électrique et mécanique aussi. Chaque mois on intervient pour remplacer et réparer. On a un marché pour changer immédiatement les toboggans et les équipements aussi. C'est un travail continu. Par contre pour les autres parcs, je parle du Grand Tunis, l'entretien n'est ni hebdomadaire ni régulier. Il s'agit d'une tâche d'urgence. Lorsque le parc ou le jardin atteint un stade de grande dégradation et que des articles de presse paraissent, c'est à ce moment-là qu'on intervient. » (Entretien avec le chef de service de l'embellissement urbain au MEDD, 2010.) 
Figure 3. Le parc El-Abidine à Carthage

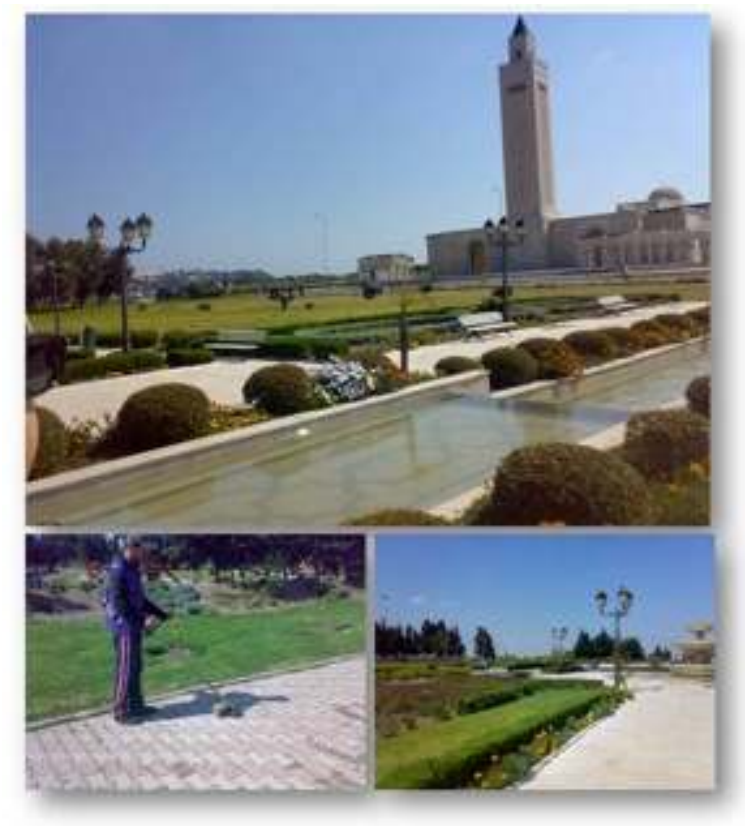

Ce parc public qui porte le nom de l'ex-président est situé sur le trajet de ce dernier, entre sa demeure privée et le palais présidentiel. II est suivi par le ministère avec une sous-traitance d'un entrepreneur privé.

Source : Besma Loukil.

24 La gestion des parcs par l'Agence nationale de protection de l'environnement constitue un cas à part. En effet, les parcs régentés par l'ANPE le sont d'une manière différente des deux précédentes institutions. Des parcs comme celui de Nahli dispose d'un directeur. Le directeur est le responsable qui connaît très bien son parc et s'en occupe. C'est à lui de déterminer et de demander un budget. C'est au directeur de l'Agence nationale de protection de l'environnement de décider le montant qui devrait être accordé, en fonction des demandes des autres directeurs de parcs. Ce directeur procède ainsi à l'élaboration d'un programme commun à tous les parcs pour pouvoir déterminer le montant nécessaire à chacun des parcs. Ce dernier est variable d'une année à une autre. Si un parc dispose d'un montant nécessaire pour l'année en cours, cela ne sera pas forcément le cas les années suivantes. 
Figure 4. Carte de la localisation des parcs et des jardins étudiés dans le gouvernorat de Tunis

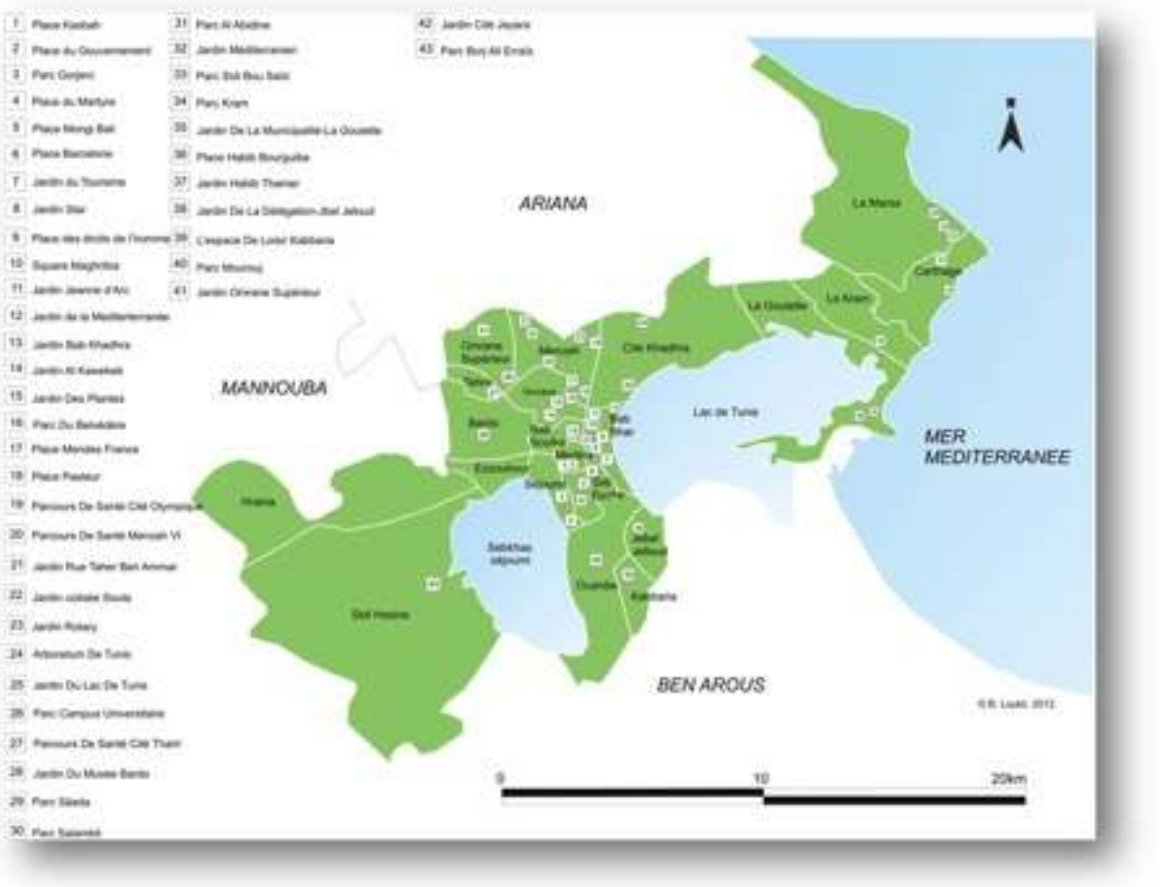

«Les parcs ont chacun un budget bien déterminé. C'est un budget pour chaque année. Un budget qui dépasse $1 \mathrm{MD}$ chaque année pour leur entretien. Les parcs Nahli et Mourouj, qui sont gérés par l'Agence nationale de protection de l'environnement, et les parcs gérés par le ministère de l'Environnement et du Développement durable sont des parcs qui possèdent chacun un responsable et disposent d'une équipe. Cependant, les autres parcs qui sont gérés par les municipalités n'ont pas de cadre de suivi. Ils ne sont sujets d'aucune planification d'entretien ou de gestion. Les parcs comme Mourouj et Farhat Hached et ceux gérés par le ministère de l'Environnement et du Développement durable ont leurs responsables. Les parcs et les jardins qui sont gérés par les municipalités sont laissés à leur propre sort. Par contre nos parcs ont tous une politique de gestion. Donc c'est l'intensité et la fréquence de gestion qui entrent en jeu. » (Entretien avec un ingénieur principal au MEDD, 2010.)

Les propos du directeur responsable des parcs urbains à l'Agence nationale de protection de l'environnement confirment que les parcs sous sa tutelle disposent d'un plan et d'un budget important par rapport aux espaces managés par les municipalités. Cependant le travail reste tributaire d'autres facteurs d'ordre politique.

Selon les parcs, les usagers ne sont pas les mêmes et le type de régie ne l'est pas non plus. Dans les quartiers aisés, l'attractivité des parcs est très faible, selon un directeur à l'Agence nationale de protection de l'environnement. Pour attirer les habitants, l'Agence donne l'administration des espaces de sport au privé (cas du parc Nahli dans le gouvernorat de l'Ariana). Dans les parcs publics, on voit ainsi naître des clubs dirigés par des acteurs privés et qui sont à entrée payante. La direction du parc bénéficie ainsi des montants de location des clubs et c'est à l'investisseur d'imposer des droits d'entrée. Les gestionnaires voient dans cette manière de faire une solution pour animer et faire vivre le parc par des activités nécessitant des budgets importants.

$\mathrm{Au}$ parc Mourouj, où les usagers relèvent de catégories sociales moyennes voire pauvres, la pression est forte sur les aires de jeux et sur les terrains de sport. Les 
terrains de basket sont dégradés assez souvent, les terrains de football aussi. Les gestionnaires décident alors d'arrêter leur utilisation par le public.

Les parcs régentés par les municipalités ont des moyens financiers très variables. Dans la ville de Tunis, les parcs et les jardins sont sous la responsabilité de la direction des parcs et des jardins de la ville et disposent d'un nombre important de techniciens horticulteurs et paysagistes. Les ouvriers permanents sont en nombre important. Ce qui importe le plus c'est que, du fait de la hiérarchie qui domine au sein de ces services, le rôle de l'ouvrier est limité à l'exécution. Cependant, la présence des ouvriers permanents et celle d'ingénieurs et de techniciens qui gèrent l'équipe ajoute beaucoup de qualification aux travaux réalisés. Ce cas de figure correspond au parc du Belvédère, mais il n'est pas extensible à la totalité des parcs et des jardins publics gérés par l'État en dehors de la ville de Tunis.

Les budgets des municipalités sont, on l'a dit, très faibles. Ceci s'aggrave lorsque le parc est situé dans un environnement urbain caractérisé par un taux élevé de chômage et de criminalité (cas du parc du Kram), mais aussi dans les centralités urbaines où un taux de fréquentation élevé complique davantage les tâches de gestion (jardin Habib Thameur, parc du Belvédère). Les parcs de la ville de Tunis disposent d'un avantage c'est qu'ils comprennent un nombre important d'ouvriers de jardinage et de gardiennage. Un grand parc comme celui du Belvédère dispose de son technicien et de 76 ouvriers, dont 11 municipaux, c'est-à-dire des permanents et 65 occasionnels (entretien avec le gestionnaire du parc du Belvédère, juillet 2010). Pour les autres villes, les parcs et jardins font partie des espaces publics de la ville et sont administrés assez souvent par des non-spécialistes (ingénieurs de génie civil, responsables des travaux publics, etc.). «Moi je m'occupe de tous les espaces verts de la ville du Kram: les espaces verts et les giratoires du côté de Kram ouest. Mes ouvriers procèdent au nettoyage de ces espaces par zone. L'entretien total du parc nécessite deux jours pour le nettoyage et l'enlèvement des mauvaises herbes. Ma tâche est d'assurer la propreté de tous les espaces. Le travail se fait d'une façon cyclique. On passe un à deux jours ici dans le parc et puis on passe les autres jours dans les autres espaces verts. » (Entretien avec l'entrepreneur du parc du Kram, juillet 2010). Le renouvellement trop rapide du personnel est une autre explication. En ce qui concerne le gardiennage, il s'avère insuffisant surtout dans les quartiers populaires.

\section{Ce qu'apporte la coopération internationale : le cas du Jardin méditerranéen}

30 La coopération internationale se matérialise par une collaboration financière dans la majorité des cas. Dans le cas du Jardin méditerranéen à Sidi Bou Saïd, elle est aussi technique: conception, exécution, suivi et financement. À cause de l'intervention étrangère (La Principauté de Monaco), le Jardin méditerranéen constitue un espace qui bénéficie d'un traitement particulier dans la ville. En comparant les travaux d'exécution réalisés dans le Jardin méditerranéen à ceux réalisés dans le parc de Sidi Bou Saïd, le technicien explique que l'écart ne vient pas seulement des contraintes financières, il est dû aussi aux contraintes liées au degré de confiance donné par les administratifs aux techniciens, ingénieurs, concepteurs et ouvriers. En effet le rôle de la Principauté de Monaco n'a pas été seulement important pour la création mais aussi dans le maintien du jardin en bon état. En effet, cet espace bénéficie du soin du prince 
de Monaco en personne. Il rend visite au parc chaque fois qu'il visite la Tunisie. Une équipe technique vient périodiquement pour le contrôler.

«En plus on a le plan de masse, qu'on est en train d'appliquer. C'est-à-dire le jardinier et son chef ne doivent rien modifier par rapport aux plantes qui sont sur le plan. Ils doivent suivre le plan à la lettre, respecter la palette végétale et les symétries [...]. Les charges de l'entrepreneur sont payées par Monaco [...] Monaco paye chaque année presque 30000 DT. Ce montant sert à payer les 2 ouvriers, leur chef et les frais d'achat des manquants [...]. Le groupe de travail de Monaco exige l'application du plan-masse. Il sollicite même les méthodes de taille [...] Le matin on trouve le gazon en état dégradé, mais en l'irriguant il reprend vie. Tu sais pourquoi le gazon fait une reprise rapide? Parce que tout simplement cela revient au travail de l'équipe. Ils ont fait tout le travail dans les règles de l'art. (Entretien avec le technicien de la municipalité de Sidi Bou Saïd.)

La coopération internationale apporte un avantage pour l'étude, l'aménagement, l'exécution et l'entretien du projet paysager du moment. Elle permet avant tout une vision de recul et une comparaison des moyens et du savoir-faire mis en place pour la création et le maintien d'un jardin public. Car, hors de ce cas particulier, des décalages financiers mais aussi des différences de technicité et de qualification, un manque de maîtrise et de prise de décision des ingénieurs et des techniciens de l'aménagement paysager sont souvent observés

La plupart du temps, les paysagistes fonctionnaires, les architectes chefs de service et les urbanistes ont à exécuter la stratégie et les décisions de l'État. Le chef de service des espaces verts au ministère de l'Environnement et du Développement durable le confirme :

«Lorsqu'on reçoit une volonté politique..., même si on est ministre de l'Environnement, on ne peut pas dire non. Il s'agit d'une instruction. J'ai tant détesté ce terme : «taaliimaat » (« instructions »). C'est une instruction : on ne peut rien faire. Même si je suis ministre de l'Environnement, je ne peux rien faire. Il faut se mettre à la place des gens. Personnellement je ne peux pas dire non, c'est un projet présidentiel, c'est des instructions présidentielles. » (Entretien réalisé après la Révolution tunisienne, février 2011).

\section{Intérêt et limites des entreprises privées}

L'intérêt de la régie mixte ou privée dans la gestion des parcs et des jardins publics est que ces modes assurent le maintien de l'espace. L'entreprise privée dispose d'une liste de tâches à effectuer, et c'est aux institutions publiques de contrôler l'avancement des travaux. Dans ce cas de figure, les dégradations sont vite réparées, les plantes arrachées sont rapidement remplacées, la présence quotidienne des ouvriers de jardinage et de propreté renforce le contrôle de l'espace, en présence qu'il y ait un gardien (parc Abidine à Carthage, parc Sidi Bou Said) ou pas (cas de la place Pasteur, jardin Jeanne d'Arc). Ceci est vrai pour le Nord-Est de la Tunisie. Les entreprises sont d'une manière générale celles des paysagistes, des horticulteurs ou des pépiniéristes en Tunisie. La gérance se fait également suivant une convention entre les municipalités et des entreprises privées dont le chef n'est pas nécessairement spécialisé. Les ouvriers également ne sont pas toujours qualifiés. On se trouve donc souvent dans une situation où toute l'équipe (cas du parc du Kram) est non spécialisée. Certaines conventions ont été réalisées dans l'objectif de créer de l'emploi pour les diplômés. Ce qui rend la 
situation plus critique c'est que ces ouvriers mal payés ne restent pas longtemps et n’apprennent pas le métier.

« [...] L'essentiel c'est la maîtrise de l'équipe des ouvriers. La mentalité des ouvriers est très différente de la nôtre (rire), c'est une grande différence. La difficulté réside dans le fait que c'est une catégorie un peu difficile ; surtout lorsqu'on est des cadres et qu'on est habitué à se comporter avec des ouvriers. Il faut savoir s'imposer avec les ouvriers, il faut savoir comment les maitriser. Il y a des périodes où on doit les gâter, sorte de récompense, et des fois non. [...] Assez souvent, ils ne sont pas sérieux et je suis amenée à les virer pour qu'ils ne puissent pas influencer négativement les autres membres de l'équipe. Je suis obligée de virer certains ouvriers pour faire peur aux autres. C'est aussi le problème de la municipalité. Cette dernière souffre aussi des comportements des ouvriers. C'est notre problème à tous.» (Interview d'une entrepreneure.)

Il ne faut pas oublier que les ouvriers ne sont pas assez bien payés comparativement aux cadres. Dans ce sens la vision de l'entrepreneure reste discutable. D'ailleurs cette dernière ne nous a pas permis de les rencontrer.

Les municipalités cèdent certains jardins à des grandes sociétés. Ceci s'effectue sans changer le statut foncier de l'espace (jardin Star). Dans ce cas, les sociétés assurent l'entretien et l'espace reste ouvert au public selon les horaires administratifs. Les municipalités donnent aussi la régie en partie à des personnes ou à des entreprises privées qui s'occupent d'un kiosque, d'une buvette, d'un minizoo, d'une aire de jeux, de terrains de sport, etc. L'espace environnant ces composantes reste alors sous la surveillance de l'investisseur privé (parc Ben Arous et, depuis fin 2010 le parc du Kram), mais le reste de l'espace demeure sans gestionnaire ou sous la régie de la direction des forêts dans le cas des grands parcs, en partie boisés. Dans ce cas de figure l'entrée peut rester gratuite mais les services sont payants. Ce mode de gestion est généralisé dans le Nord-Est de la Tunisie (Menzel Temime).

«... la municipalité de Menzel Temime a donné tous ses espaces verts aux privés qui gèrent des buvettes. L'espace est ouvert même le soir et les familles pourraient $\mathrm{y}$ passer des moments agréables. Et, en parallèle, le privé s'occupe du maintien de l'espace qui reste en bon état. Comme je t'ai déjà expliqué, les municipalités ne pourraient jamais consacrer un grand budget pour les espaces verts. » (Entretien avec le chef de service de l'embellissement urbain au MEDD, juin 2010)

En dehors de la privatisation de la gérance de ces parcs, certains parcs sont devenus privés (entrée payante et services payants : parc Saada à la Marsa) ou sont fermés aux publics depuis des années (parc Tadhamen). Les parcs fermés ou privés ne peuvent ni assurer la fonction d'espaces publics accessibles à tous sans aucune distinction, ni, comme en Europe, " contribuer à la fois à pacifier les relations sociales et à développer le sentiment d'appartenance des individus à une même "cité" ». (Levy et Lussault, 2003).

\section{Conclusion}

Les parcs publics de la région de Tunis sont soumis à plusieurs modes de gouvernement qui supposent des budgets très différents avec des résultats satisfaisants à discutables. Une partie est sous la tutelle de l'État. La majorité est prise en charge par les municipalités et dispose de moyens financiers très variables selon la nature des quartiers concernés : centraux, populaires ou aisés. Selon les quartiers, des différences importantes ont été enregistrées et illustrent bien les types de politique de gestion. Le maintien d'une partie des parcs et des jardins publics, comme celui des services que le 
public y trouve, est cependant de plus en plus confié à des entreprises privées. Cela donne aux gestionnaires des espaces publics des moyens financiers et des modes d'intervention réguliers qui aident à réparer les dégradations des équipements et de la végétation.

Cependant, la privatisation de ces espaces et l'administration des équipements de jeux ou de loisirs limitent l'accès de tous à ces espaces publics. Donc il faut distinguer la privatisation des usages de l'espace de celle de son entretien. Dans certains cas, comme le Jardin méditerranéen de Sidi Bou Saïd, la contribution financière de la coopération étrangère permet d'obtenir des résultats très satisfaisants grâce au suivi attentif d'un plan prédéfini, tout en gardant l'espace accessible à tous sans aucune distinction. Les disparités enregistrées concernent également le contrôle formel de ces espaces publics. Dans les quartiers où l'on enregistre plus de chômage et de délinquance, on trouve moins de contrôle et moins d'interventions des gestionnaires.

Nous tenons à remercier tous les acteurs publics ayant accepté de répondre à nos questions avant la Révolution tunisienne, période où les déclarations qu'ils nous faisaient pouvaient leur nuire.

\section{BIBLIOGRAPHIE}

Barthe-Deloizy, F., « Parcs et jardins : étude de pratiques spatiales urbaines (Les Mureaux-SaintGermain-en-Laye) », thèse de doctorat en géographie, UFR de géographie, Paris IV Sorbonne, 1997, 2 tomes, $361 \mathrm{p}$.

Ben Ayed-Najjar, R.,2007, « Une mosaïque d'ambiances. Scénographie à la place Bab Bhar à Tunis ", dans Thibaud, J.-P., Variations d'ambiances, processus et modalités d'émergences des ambiances urbaines, Grenoble, Laboratoire Cresson, octobre 2007, 275 p., p. 85-113.

Ben Jbara, S., « Ambiances et intégration urbaine d'un espace paysager public : cas du jardin Habib Thameur ", mémoire de master en urbanisme ENAU, 2006, 179 p.

Boudon, R., et Bourricaud, F., Dictionnaire critique de la sociologie, Paris, Presse universitaire de France, 2004, $713 \mathrm{p}$.

Bouraoui, M., «L'agriculture, nouvel instrument de la construction urbaine ? Étude de deux modèles agri-urbains d'aménagement du territoire : Le plateau de Saclay, à Paris, et la plaine de Sijoumi, à Tunis ", thèse de doctorat en sciences de l'environnement, soutenue à l'Engref, Paris, $2000,442 \mathrm{p}$.

Bretzel, F., et Pezzarossa, B., " Sustainable management of urban landscapes with wildflowers ", Acta Horticulturae, $\mathrm{n}^{\circ}$ 881, editions ISHS, 2010.

Campeau, R., Sirois, M., Rheault, E., Dufort, N., Individu et Société. Introduction à la sociologie, Montréal, Gaëtan Morin Éditeur, 1998, 433 p.

Dictionnaire de la sociologie, Encyclopædia Universalis, Paris, Albin Michel, 1998, 922 p.

Donadieu, P. et Mazas E., Des mots de paysage et de jardin, Dijon, Educagri éditions, Dijon, 2002, 317 p. 
Ghorra-Gobin, C., «L'utopie de la ville au Xxi ${ }^{\mathrm{e}}$ siècle : entre nouvelle donne politique et mythes fondateurs ", rencontres sur l'utopie : " Peut-il y avoir une utopie de la ville? », publié en mai 2000, URL: http://www.abhatoo.net.ma/index.php/fre/Maalama-Textuelle/ D\%C3\%A9veloppement-\%C3\%A9conomique-et-social/D\%C3\%A9veloppement-social/Logementet-habitat/Logement-et-habitat-G\%C3\%A9n\%C3\%A9ralit\%C3\%A9s/L\%E2\%80\%99Utopie-de-laville-au-XXIe-si\%C3\%A8cle-entre-nouvelle-donne-politique-et-mythes-fondateurs/ \%28language\%29/fre-FR, consulté en 2008, dernier accès janvier 2012.

Jaballah, A., « Le parc du campus universitaire El-Manar », mémoire de master en paysage, territoire et patrimoine, ISA Chott Mariem, Sousse, 2006, 120 p.

Jacob, J., The Death and Life of Great American Cities, New York, Random House, 1961; Déclin et survie des grandes villes américaines, traduit en français par C. Parin-Sénemaud, Wavre, Mardaga, (cité par Wyvekens, 2007).

Joshi, T. et al., « Urban Landscape Management and Implications: for Greening of Urban Areas and Controlling Pollution in Context to Indian Conditions ", Acta Horticulturae, $\mathrm{n}^{\circ}$ 881, éditions ISHS, 2010, p. 235-239.

Le Nouveau Petit Robert de la langue française, Paris, 2008, 2838 p.

Lévy, J. et Lussault, M. (sous la dir.), Dictionnaire de la géographie et de l'espace des sociétés, Paris, Belin, 2003, 1033 p.

Loukil, B., «Les nouveaux parcs urbains à Tunis, pour qui et pourquoi ? Étude de trois parcs urbains au grand Tunis ", mémoire de mastèer en Paysage, territoire et patrimoine, ESHE, Chott Mariem, 2006, 129p.

Merlin, P., Choay, F. (1988), Dictionnaire de l'urbanisme et de l'aménagement, Paris, Presse universitaire de France, 4e édition, 2005, 963 p.

Morgan, D., Newell, R., Pitcher, F., « Reduce crime and disorder in Park », Problem solving quarterly, vol. 8, $\mathrm{n}^{\circ} .314,1995$, p. 1-4.

Newman, O., Defensible space: people and design in the violent city, London, Architectural press, 1972, 264 p., (cité par Wyvekens, 2007).

Le Nouveau Petit Robert, 2001, Paris, Dictionnaires le Robert, 2841 p.

Nugier, A. et al., « Déviance, émotions morales, et contrôle social informel : le rôle des émotions dans la régulation des comportements contre normatifs ", séminaire invité au laboratoire de psychologie sociale de l'université Paris 5, Paris, France, 2007.

Parsons, T., « Le contrôle social », dans Dictionnaire de la sociologie, Paris, Encyclopædia Universalis, Albin Michel, 1998, 922 p.

Randrup, T-B. et al., «Public Green Spaces in the Nordic Countries: Development of a New Strategic Management Regime », Urban Forestry \& Urban Greening, vol. 8, issue 1, 2009, p. 31-40.

Turki, S.Y., Zhioua, I., « Les espaces verts dans le Grand Tunis, entre programmation et réalisation ", dans Turki, S.-Y., Villes et Espaces verts, Tunis, Centre de publication universitaire, 2006 ; actes du séminaire organisé à l'Institut supérieur des technologies de l'environnement, de l'urbanisme et du bâtiment, nov.-déc.-2006, p. 65-86, 150 p.

Wilson, J.-Q., et Kelling, G.-L., The police and neighborhood safety, broken windows, 10 p., 1982, URL : http://www.manhattan-institute.org/pdf/_atlantic_monthly-broken_windows.pdf, consulté en mars 2007, dernier accès décembre 2011. 
Zaâfrane Zhioua, I., « Le parc du belvédère de Tunis, un siècle d'histoire ", mémoire de DEA, Jardins, paysages, territoires, à l'école d'architecture Paris-La Villette, 1998.

Zahaf, L., " Pour une deuxième vie au belvédère ", mémoire de troisième cycle en architecture, École nationale d'architecture et d'urbanisme, 1999, 78 p.

\section{NOTES}

1. La thèse est intitulée «Civilités et incivilités dans les parcs et les jardins publics au nord-est de la Tunisie, interactions entre gestionnaires et pratiques sociales».

2. Cas des quartiers comme Kabbaria, Ouardia, Tadhamen classés comme quartiers populaires (Bouraoui, 2000).

3. Régie directe: espaces gérés directement par des institutions de l'État (MEDD, ANPE, municipalités); régie mixte: espaces gérés par les institutions étatiques et des entreprises privées d'entretien et de propreté : régie indirecte : espaces gérés par les entreprises privées.

4. Nous sommes dans l'impossibilité de fournir des chiffres concernant les budgets alloués aux municipalités des villes tunisiennes. Ces chiffres sont la propriété du ministère de l'Intérieur et du Développement local et ne sont pas fournis. Notre seule source est celle des interviews avec les acteurs publics.

\section{RÉSUMÉS}

Dans cet article, nous cherchons à définir les notions de d'entretien, de contrôle des espaces publics de type parcs et jardins, et à préciser les raisons qui permettent d'interpréter les variations observées dans le territoire du Nord-Est de la Tunisie. Il traite en premier lieu les contraintes rencontrées par les acteurs de la gestion, entre autres ceux en rapport avec les comportements des usagers. Ensuite, il illustre le rôle de l'État dans la mise en œuvre, directement à travers le financement et indirectement à travers sa politique de création et de répartition, de ces espaces au sein de l'environnement urbain. Enfin, le présent texte montre ce qu'apportent les entreprises privées et la coopération internationale à la qualité de l'intervention dans la gestion des parcs et des jardins publics ainsi que les défaillances induisant une dégradation de l'entretien.

In this paper we are explaining the noticed fluctuations in management of public spaces such as urban parks. First of all, we treat the constraints met by the actors of the management. Some constraints are due to the user's behavior. Then, we illustrate the role of the public policy and its effect on management practices directly through the financing and indirectly through creation and distribution of these spaces within the urban environment. Finally, the present text shows the adding value of private companies and international cooperations to the quality of the contribution in the management of parks and public gardens. 
INDEX

Keywords : parks and gardens, public space, management, maintenance, control, public policy, Tunisia

Mots-clés : parcs et jardins, espace public, gestion, entretien, contrôle, politique publique, Tunisie

\section{AUTEURS}

BESMA LOUKIL

Ingénieur paysagiste, elle est doctorante en cotutelle à AgroParisTech et à l'Institut supérieur agronomique de Chott Mariem à Sousse.

loukilb2004[at]yahoo[dot]fr

\section{TAOUFIK BETTAIEB}

Maître de conférences à l'Institut national agronomique de Tunis.

tbettaieb[at]yahoo[dot]fr

\section{PIERRE DONADIEU}

Professeur à l'École nationale supérieure de paysage de Versailles-Marseille.

p.donadieu[at]icloud[dot]com 